\title{
Recent developments in density functional approximations
}

\author{
Li Li and Kieron Burke
}

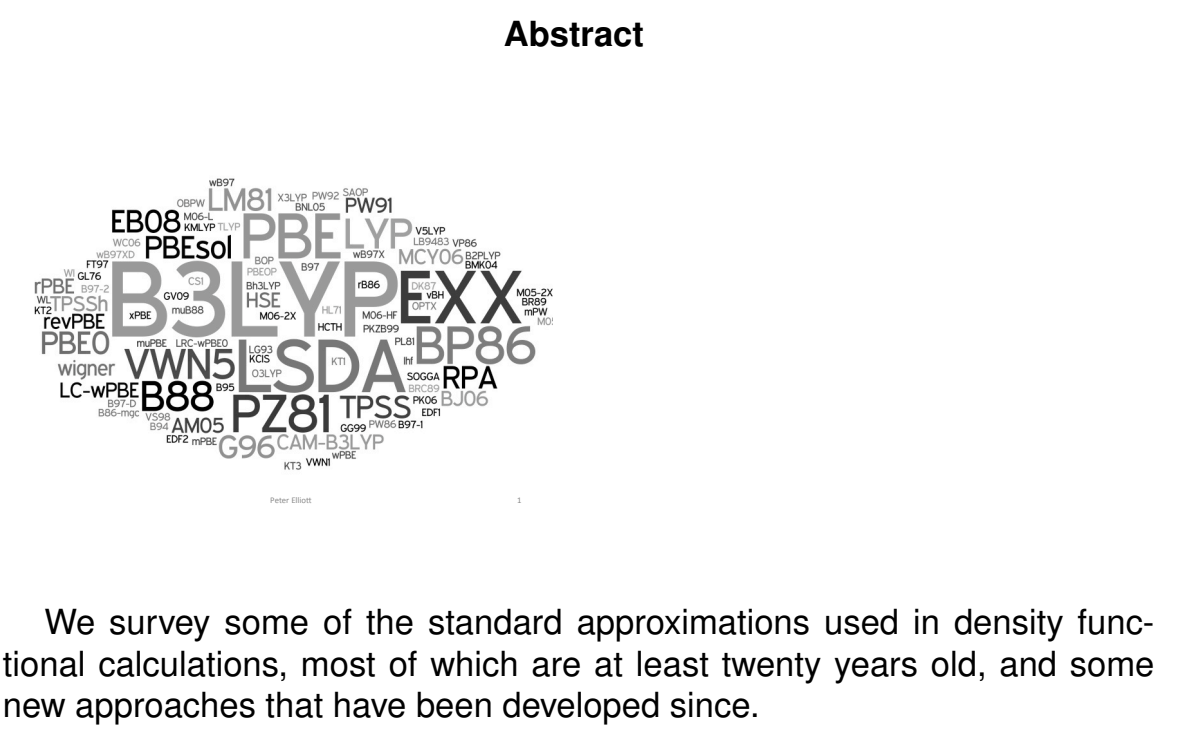

\section{Introduction}

Each year, at least 30,000 papers are published using density functional theory to perform electronic structure calculations (Pribram-Jones et al 2015).

$\mathrm{Li} \mathrm{Li}$

Department of Physics and Astronomy, UC Irvine. Now at Google. e-mail: li. li@uci .edu

Kieron Burke

Departments of Physics and of Chemistry, UC Irvine, e-mail: kieron@uci.edu

To appear in Handbook of Materials Modeling, Vol 1, Methods: Theory and Modeling, 2018 


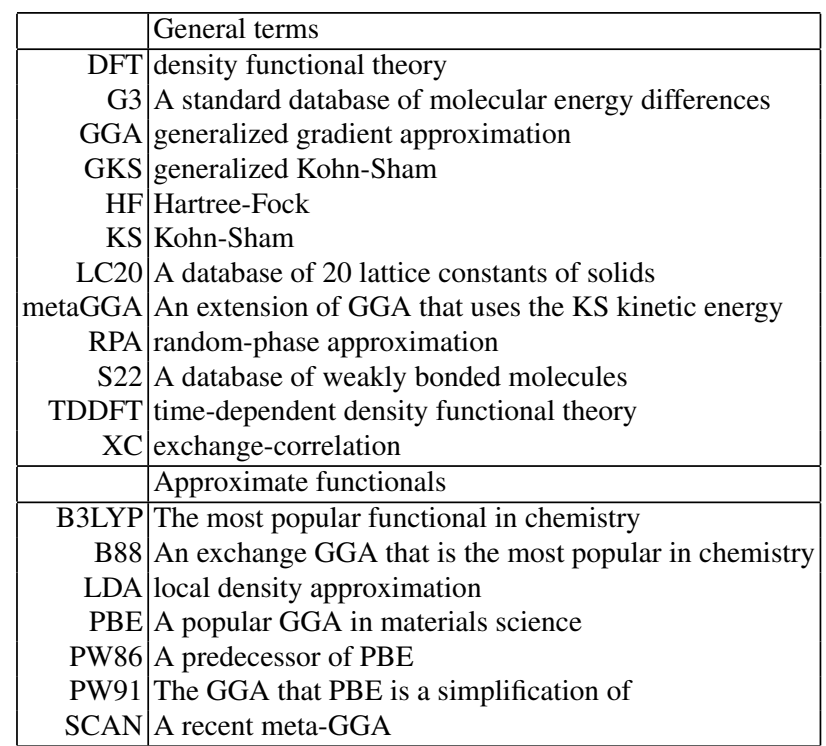

Table 1 Table of acronyms in alphabetical order. References are given where they first appear in main text.

Almost all solve the Kohn-Sham (KS) equations (Kohn and Sham 1965) selfconsistently, and use some approximation for the exchange-correlation (XC) energy as a functional of the (spin)-densities, $E_{\mathrm{XC}}[n]$. This article surveys some of the more popular approximations. Some background is covered in Burke (2012), which is more focused on chemistry.

\subsection{Commonly used approximations}

The original approximation was suggested by Kohn and Sham themselves (Kphn and Sham 1965), namely the local density approximation (LDA), in which the XC energy density at each point in the system is replaced by that of a uniform electron gas with the density at that point. The exchange contribution was first written by Bloch (Bloch 1929), with correlation now well-known from quantum Monte Carlo simulations of the uniform gas (Ceperley and Alder 1980), parametrized in simple formulas (S. H. Vosko and Nusair 1980; Perdew and Wang 1992). LDA is remarkably accurate for geometries, but typically overbinds molecules by about $1 \mathrm{eV}$ per bond, making it relatively useless for thermochemistry (Jones and Gunnarsson 1989).

The next step in complexity is the generalized gradient approximation (GGA), which creates an energy density using both the density and its gradient at each point (Burke et al 1997a). The basic concept, and its first real- 
ization, were given already in the pioneering work of Ma and Brueckner (Ma and Brueckner 1968). This was carefully refined in the work of Langreth, leading to the Langreth-Mehl functional (Langreth and Mehl 1981, 1983). Perdew pioneered the use of real-space cutoffs to create GGA's, leading to the PW86 functiona Perdew and Wang (1986). The highpoint of this detailed construction was the PW91 functional (Perdew 1991; J. P. Perdew and Fiolhais 1992; Burke et al 1997b), and in 1993, it was shown that they yield useful accuracy for binding energies, i.e., errors of about $6-10 \mathrm{kcal} / \mathrm{mol}(1$ $\mathrm{eV}=23 \mathrm{kcal} / \mathrm{mol}$ ) (Gill et al 1992). The most commonly used GGA in materials today is a simplification of the PW91 form called PBE (Perdew et al 1996a), while in chemistry it is BLYP, with B88 exchange (Becke 1988) and Lee-Yang-Parr correlation (Lee et al 1988). Both these are trained on Noble gas atomic energies, yielding more accurate energies for those atomsElliott and Burke (2009); Burke et al (2016).

The last standard step is to create a hybrid of GGA with the exact exchange energy from a Hartree-Fock calculation, by replacing a fraction, $a$, of the GGA exchange with the Hartree-Fock (HF) exchange, as first suggested by Becke (Becke 1993). This fraction is 20\% in the famous B3LYP functional, (which stands for Becke, 3-parameter, Lee-Yang-Parr), the most commonly used approximation in chemistry today (Becke 1993; Lee et al 1988; S. H. Vosko and Nusair 1980; P. J. Stephens and Frisch 1994). Its analog is PBE0 (Perdew et al 1996b; Ernzerhof and Scuseria 1999) which uses $25 \%$ mixing.

At least $80 \%$ of all DFT calculations currently being performed use one of the approximations mentioned above. One can think of more sophisticated approximations using more ingredients of the density, as in Jacob's ladder Perdew and Schmidt (2001). An important principle of progress in making density functional approximations is to ensure that (almost) everywhere, each level of approximation performs at least as well as the previous level, and also improves some features, making the previous level obsolete (except to save computational time). One should avoid having different functionals for different purposes.

\subsection{Beyond ground-state DFT}

What has been described above is generic ground-state DFT in the nonrelativistic limit and for collinear magnetic fields. The single largest use of DFT beyond this domain is using time-dependent DFT (TDDFT) (Runge and Gross 1984; Maitra et al 2004; Maitra 2016) in the linear response regime to extract electronic transition frequencies (Casida 1996; M. Petersilka and Gross 1996). As much as $10 \%$ of all DFT publications include TDDFT estimates of excitations. Such calculations almost all use the adiabatic approximation for the XC kernel, and so are uniquely determined by 
the choice of ground-state approximation. Most such calculations are for molecules (Burke et al 2005), as a non-local contribution is needed to yield details of bulk semiconductor spectra correctly (such as exciton binding energies), and that contribution is missing from the commonly-used functionals mentioned above (Onida et al 2002; Martin et al 2016).

Other extensions include magnetic DFT for non-collinear fields, relativistic DFT (Engel and Dreizler 2011) and even QED (Flick et al 2015), DFT at finite temperatures using the Mermin theorem (Smith et al|2017), coupling between nuclei and electrons (Requist and Gross 2016), and so on. Again, standard ground-state approximations are used unless a particular effect requires going beyond these, such as extracting double excitations (Maitra et al 2004).

\section{Recent developments}

This section is devoted to developments over the past twenty years.

\subsection{RPA-type functionals}

The famous adiabatic-connection fluctuation-dissipation formula of DFT yields $E_{\mathrm{XC}}$ in terms of the a frequency and coupling-constant and spatial integral over the density-density response function (Langreth and Perdew 1975; Harris and Jones 1974; Gunnarsson and Lundqvist 1976). This can be extracted directly from the KS response function, constructed from the occupied and unoccupied orbitals, and the XC kernel of TDDFT (Gross and Kohn 1985). Ignoring the kernel yields RPA, also known as direct random-phase approximation (RPA) (Furche 2001; Langreth and Perdew 1977, 1975; Chen et al 2017), also known as TD-Hartree. This scheme thus yields a fifth-rung (in Jacob's ladder) approximation that can be costly to evaluate, but the relative burden is always decreasing (Furche 2008; Eshuis et al 2010).

Direct RPA overcorrelates systems, because it includes only 'bubble' diagrams in the many-body expansion of the energy, and misses other contributions at higher-order that reduce correlation. It also has difficulties with self-interaction, because it yields finite correlation energies even for only one electron. These two effects yield inaccuracies in the dissociation energies of molecules. Recent progress has included various approximations to the XC kernel to yield improved energetics and computational cost-savings. A very recent development, using the exact frequency-dependent exchange kernel, and a clever and physically motivated resummation of higher-orders, appears to overcome stability problems and even allows the binding curve of $\mathrm{N}_{2}$ to be accurately calculated (Erhard et al 2016; Burke 2016) (which is 
difficult even in coupled-cluster theory, due to the multireference nature of the wavefunction at large separations).

\subsection{Weak interactions}

Tremendous progress has been made in the last two decades for including weak, van der Waals interactions (vdW) into DFT calculations. The standard functionals model covalent, ionic, and metallic bonds reasonably well, but fail for longer, weaker bonds. Because of their semilocal nature, they cannot yield weak-binding that falls off as $R^{6}$, where $R$ is the separation between two atoms or molecules. Thus corrections must be added to the standard functionals to capture these effects. There are three main schemes for doing this.

In the domain of explicit non-local density functionals, there is the sequence of approximations originally developed by Langreth and Lundqvist and co-workers (Dion et al 2004). These approximations are derived nonempiricially, remarkably starting from contributions to the uniform gas correlation energy. The original was from 2004, and an improved parameterization was given in vdW2 (Klimeš et al|2009). These explicit density functionals can be applied to all materials, from molecules to solids, but have been designed assuming the system has a gap (not a metal) (Berland et al 2015).

At the extreme opposite end of the scale, there are the parametric schemes of Grimme and co-workers (Grimme 2006), commonly referred to as DFT-D. These are not explicit density functionals, but rather additional energies that include estimates of the $C_{6}$ (and higher-order) coefficients in the asymptotic expansion of the vdW energy between atoms, combined with a damping factor to keep the contributions finite as the separation reduces. Such schemes require empirical parameters for each atom for a given standard XC approximation, but can yield highly accurate energy curves for small weakly bound molecules (Burns et al 2017).

In between is the scheme developed by Tkatchenko and Scheffler, which requires only one parameter for a given XC functional, and produces accurate add-on corrections to DFT energies (Tkatchenko and Scheffler 2009). This has been expanded to incorporate collective electrostatic effects so that metals and materials in many dimensions and on different length scales can be treated (Hermann et al|2017). A final scheme is that begun by Becke and Johnson, which uses the dipole moment of the exchange hole to determine $C_{6}$ (and higher) coefficients (Johnson and Becke 2006).

Of course, more expensive treatments, such as RPA mentioned above, automatically include approximations to the VdW forces. 


\section{3 meta-GGA's}

The third rung of Jacob's ladder is the meta-GGA, which adds a new ingredient beyond that of the density and its gradient. This is most often chosen to be the (positive) kinetic energy density of the KS orbitals. The aim for a good meta-GGA is to aim for the accuracy of hybrids without the computational overhead of the exact exchange contribution. The cost of exact exchange is relatively manageable in molecular calculations with atom-centered basis functions, but can often be prohibitive when using periodic boundary conditions and plane-wave basis sets. Running a range-separated hybrid (see next section) can sometimes take 1000 times longer than a typical GGA in a materials calculation.

Perdew and co-workers and many others have spent several decades developing meta-GGA's, with many flawed attempts (Sun et al 2015). But the most recent effort, called strongly constrained and appropriately normed semilocal density functional (SCAN), has passed many standard tests and appears very promising to join the pantheon of commonly-used functionals. The G3 dataset (L.A. Curtiss and Raghavachari 2005) is a standard set of chemical bonds that LDA overbinds by about $3 \mathrm{eV}$ (typically about $1 \mathrm{eV} /$ bond). PBE reduces this to about $1 \mathrm{eV}$, while SCAN reduces this to about $1 / 4 \mathrm{eV}$. SCAN also has errors that are 2-3 times smaller than PBE on the S22 dataset (Jurecka et al 2006) of weakly bonded systems. At the same time, SCAN reduces errors in lattice parameters on the LC20 data (Sun et al 2011) set from about 0.05 Åin PBE to about $0.01 \AA$. SCAN also yields better water properties than PBE (Perdew et al 2017). On the other hand, the underestimation of chemical barrier heigths by PBE is only mildly improved, by about a factor of $30 \%$ (whereas hybrids are often 2-3 times better). Thus, for many properties, SCAN yields accuracies similar to hybrid functionals, but at a fraction of the computational cost (for materials codes).

\subsection{Range-separated hybrids}

The theory behind range separation is an exact one, developed first by Andreas Savin (Toulouse et al 2009; Savin 1996; Leininger et al 1997). One simply writes the Coulomb repulsion as a sum of a short-ranged contribution (decaying more rapidly than the inverse of the separation) and a longranged contribution, which has no Coulomb singularity at zero separation. One can then include one contribution as an interaction in some generalization of the KS equations, and have the redefined XC contribution accounting for the other. This is all formally exact, and exact XC functionals exist for such schemes (though they differ from their regular KS counterparts).

But a plethora of choices now await. The first is the length scale on which the range separation is performed, often denoted $1 / \omega$. This is a continuous 
parameter, and since approximations will be made to the corresponding $E_{\mathrm{XC}}$, the results are sensitive to it. Just like the fraction of exact exchange in global hybrids, there is always a temptation to adjust it.

Furthermore, the separation into long- and short-range contributions can occur for the exchange contribution (most common), the correlation, or both. Finally, one may wish to treat the short-range contribution with an approximate functional or, in other situations, the long-range contribution! For example, a vexing problem in TDDFT is to accurately calculate charge-transfer excitations of well-separated donor-acceptor complexes. Including long-range Fock exchange exactly works very well for this problem Stein et al (2009). On the other hand, the very successful HSE06 functional (Heyd et al 2003) is a range-separated hybrid, in which the long-range exchange is treated with an approximate functional, but the short-range exchange is treated exactly, to model screening in an extended insulator (Janesko et al 2009). This particular hybrid typically yields accurate gaps for moderate-gap semiconductors and insulators (Perdew et all2017).

\subsection{Gaps of solids}

A crucial failure of the standard approximations is their inability to predict gaps of semiconductors and insulators. The LDA underestimates the gap of bulk Si by a factor of 2, and makes Ge a metal, and GGA's fare little better. From very early on, a great strength of the GW method (Aryasetiawan and Gunnarsson 1998) has been its ability to provide accurate and reliable gaps.

To understand this issue, it is important to first note that the KS gap of a periodic solid does not match the fundamental gap of the solid (Perdew et al 1982; Perdew 1985). All indications suggest that in fact LDA and GGA yield reasonably accurate KS gaps (ie close to the KS gap found with the exact ground-state functional) (M. Grüning and Rubio 2006). But, unlike LDA and GGA, the exact functional allows access to the fundamental gap, which is just $I-A$, the difference between the ionization energy and electron affinity of a system. Consider a very large but finite cluster of material. One can then add and subtract an electron to find $I$ and $A$ and deduce the exact gap. In fact, modern methods exist for doing this in a periodic calculation (M. Stadele and Vogl 1999). But in LDA or GGA, the added electron or hole delocalizes over the entire system and, because of their lack of a derivative discontinuity, $I-A$ collapses to the HOMO-LUMO KS energy difference, i.e., the KS gap.

A great success of the past two decades has been the accurate calculation of moderate gaps using hybrid functionals such as HSE06 (Heyd et al 2003). This is achieved by going to a generalized KS scheme (Seidl et al 1996), in which the orbital dependent part of the functional is treated as in HF theory, not pure KS theory (which would require treating it with optimized effective potential (OEP) methods (Kümmel and Kronik 2008)). By having 
an orbital-dependent potential, one can show that the generalized KohnSham (GKS) gap of such a calculation does match $I-A$ and because of the orbital-dependence, an approximate derivative discontinuity is included. Thus the GKS gap in such a calculation is the approximate fundamental gap, and is wider than the corresponding KS gap. This is how hybrid functionals and meta-GGA's yield wider and generally more accurate gaps than GGA's (Perdew et al 2017).

\section{Challenges and hopes}

Here we review some of the more depressing failures of our current approximations.

\subsection{Strong correlation}

The failure of DFT with standard approximations for strongly correlated systems has been known since before its invention, as HF has problems for $\mathrm{H}_{2}$ when it is stretched (Heitler and London 1927). The problem can be analyzed and related to localization/delocalization errors of the standard approximations when integer (or half-integer) numbers of electrons localize on different sites (Cohen et al 2008). This is often called static correlation in quantum chemistry and involves the KS gap between two states becoming very small, and the exact many-body wavefunction becoming an (almost) equal mixture of two Slater determinants.

It is important to note that the difficulties lie only with the failure of approximations under these circumstances, rather than the KS scheme itself. This can be beautifully illustrated with the two-site Hubbard model, for which it is trivial to construct the exact KS system, even when strongly correlated (Carrascal and Ferrer 2012). Even in realistic cases (albeit in 1d), the KS equations for a strongly correlated system always can be made to converge to the exact ground-energy and density if the exact XC functional is used (Wagner et al 2013). But of course many of the features of the KS system do not resemble those of the physical system under such conditions (Carrascal et al 2015). This point is often confused by practitioners of many-body theory. The differences between KS response functions and the many-body analogs for strongly-correlated systems is not a signal that a density functional approximation is failing to yield accurate energies for such systems.

But strong correlation in solids is even more difficult than static correlation in molecules. To see this simply, consider chains of uniformly spaced $\mathrm{H}$ atoms. As the spacing is increased, an electron localizes on each site. For $\mathrm{H}_{2}$, the true wavefunction combines two Slater determinants. But for 
$\mathrm{H}_{4}$, there are 4 such determinants, and a DFT calculation with, say LDA, will break symmetry into 4 different solutions, one of which will have lowest energy (Wagner et al 2014). As the number of atoms in the chain grows, so does the number of nearly degenerate solutions, separated by spin excitations of very small energy. In the thermodynamic limit, these become infinite, and the usual quantum chemical starting point of a single Slater determinant becomes hopeless (Qiu et al 2017). Because this is such an important problem, vast amounts of research have been performed studying this limit, especially by the group of Weitao Yang (Zheng et al 2011), but also by Scuseria (Motta et al 2017), and Becke (Johnson and Becke 2017).

\subsection{The role of empiricism}

The most practical systematic approach to the construction of density functionals has that been championed by Perdew: Combine exact conditions that are relevant to a given level of approximation with appropriate norms, such as the uniform gas or hydrogen atom, to create approximations of tremendous generality (Medvedev et al 2017). A key aspect of this approach is that, as one rises on Jacob's ladder, each successive approximation works better than the previous one (or at least is no worse) under almost all circumstances (and the cases where it does not are usually very informative). Thus, for a given computational cost, there is a single (or at least, very similar) obvious choice that is rarely worse than using a lower rung. Use of appropriate norms may appear empirical, but it can be understood as choosing parameters in the approximations to capture limits that have not been fully derived as yet, but in principle could be. The extremely successful B88 exchange functional (Becke 1988; Elliott and Burke 2009) can be viewed as incorporating appropriate norms (exchange energy of atoms).

On the other hand, the profusion of inexpensive computing resources has led to many databases with either experimental results or those of high-level computational chemistry against which new approximations can be tested. It has also led to empirical fitting of density functional approximations with many parameters, as championed by Truhlar and coworkers (Zhao and Truhlar 2008, 2006; Zhao et al 2006). This approach typically produces more accurate approximations than those of Perdew et al for the systems and properties fitted, and for related systems and properties. But it does not yield single universal approximations that generically improve over previous steps on the ladder. Such approximations can fail badly when applied beyond their range of applicability.

An entirely new approach to functional approximation is to use machinelearning to learn from accurate data (Snyder et al 2012; Rupp et al 2012; Bartók et al 2010). This differs from the earlier approaches, because it automatically includes highly nonlocal contributions, as captured for example 
in the kernel which measures density separations by integrals over the entire system (Snyder et al 2012; Li et al 2016b; Vu et al 2015; Snyder et al 2013). Recent advances include the first KS-MD simulation with an ML-DFT approximation to bypass solving the KS equations (Brockherde et al 2017), and accurate approximation of the full interacting functional (including XC) (Li et al 2016a) even for strong correlation and even for extended systems (but so far, only in $1 \mathrm{~d}$, because of the cost of generating accurate data).

\subsection{New horizons}

An entirely new arena for DFT has grown immensely in the last decade or so is applications to warm dense matter (Smith et al 2017), with temperatures significant on the electronic scale (about 100,000K), but not so high that Thomas-Fermi theory (or classical behavior) dominates. Applications range from modeling planetary interiors to inertial confinement fusion. This field is so 'hot' that even the input to thermal LDA, the XC energy of a uniform gas as a function of temperature, is only now being calculated at high accuracy (Groth et al 2017).

Acknowledgements Work supported by US Department of Energy under Grant DE-FG02-08ER46496.

\section{References}

Aryasetiawan F, Gunnarsson O (1998) The gw method. Reports on Progress in Physics 61(3):237, URL http://stacks.iop.org/0034-4885/61/i=3/a=0 02

Bartók AP, Payne MC, Kondor R, Csányi G (2010) Gaussian approximation potentials: The accuracy of quantum mechanics, without the electrons. Phys Rev Lett 104:136,403, DOI 10.1103/PhysRevLett.104.136403, URL http://link.aps.org/doi/10.1103/ PhysRevLett.104.136403

Becke AD (1988) Density-functional exchange-energy approximation with correct asymptotic behavior. Phys Rev A 38(6):3098-3100, DOI 10.1103/PhysRevA.38.3098, URL http://dx.doi.org/10.1103/PhysRevA.38.3098

Becke AD (1993) Density-functional thermochemistry. iii. the role of exact exchange. The Journal of Chemical Physics 98(7):5648-5652, DOI 10.1063/1.464913, URL http:// link.aip.org/link/?JCP/98/5648/1

Berland K, Cooper VR, Lee K, Schröder E, Thonhauser T, Hyldgaard P, Lundqvist BI (2015) van der waals forces in density functional theory: a review of the vdw-df method. Reports on Progress in Physics 78(6):066,501

Bloch F (1929) Über die quantenmechanik der elektronen in kristallgittern. Zeitschrift für Physik A Hadrons and Nuclei 52(7):555-600

Brockherde F, Vogt L, Li L, Tuckerman ME, Burke K, Mller KR (2017) Bypassing the KohnSham equations with machine learning. Nature Communications URL https : / / doi . org/10.1038/s41467-017-00839-3 
Burke K (2012) Perspective on density functional theory. J Chem Phys 136, URL http: //link.aip.org/link/doi/10.1063/1.4704546

Burke K (2016) Viewpoint: Improving electronic structure calculations. Physics 9(108), URL https://physics.aps.org/articles/v9/108

Burke K, Perdew JP, Wang Y (1997a) Derivation of a generalized gradient approximation: The PW91 density functional, Plenum, NY, p 81. URL http://link.springer. com/chapter/10.1007\%2F978-1-4899-0316-7_7

Burke K, Perdew JP, Wang Y (1997b) Derivation of a generalized gradient approximation: The PW91 density functional, Plenum, NY, p 81

Burke K, Werschnik J, Gross EKU (2005) Time-dependent density functional theory: Past, present, and future. The Journal of Chemical Physics 123(6):062206, DOI 10.1063/1. 1904586, URL http://link.aip.org/link/?JCP/123/062206/1

Burke K, Cancio A, Gould T, Pittalis S (2016) Locality of correlation in density functional theory. The Journal of Chemical Physics 145(5):054,112, DOI http://dx.doi.org/ 10.1063/1.4959126, URL http://scitation.aip.org/content/aip/journal/ jcp/145/5/10.1063/1.4959126

Burns LA, Faver JC, Zheng Z, Marshall MS, Smith DG, Vanommeslaeghe K, MacKerell Jr AD, Merz Jr KM, Sherrill CD (2017) The biofragment database (bfdb): An open-data platform for computational chemistry analysis of noncovalent interactions. The Journal of Chemical Physics 147(16):161,727

Carrascal DJ, Ferrer J (2012) Exact Kohn-Sham eigenstates versus quasiparticles in simple models of strongly correlated electrons. Phys Rev B 85:045,110, DOI 10.1103/PhysRevB.85.045110, URL http://link.aps.org/doi/10.1103/ PhysRevB.85.045110

Carrascal DJ, Ferrer J, Smith JC, Burke K (2015) The hubbard dimer: a density functional case study of a many-body problem. Journal of Physics: Condensed Matter 27(39):393,001, URL http://stacks.iop.org/0953-8984/27/i=39/ $a=393001$

Casida ME (1996) Time-dependent density functional response theory of molecular systems: theory, computational methods, and functionals. In: Seminario JM (ed) Recent developments and applications in density functional theory, Elsevier, Amsterdam

Ceperley DM, Alder BJ (1980) Ground state of the electron gas by a stochastic method. Phys Rev Lett 45:566

Chen GP, Voora VK, Agee MM, Balasubramani SG, Furche F (2017) Randomphase approximation methods. Annual Review of Physical Chemistry 68(1):421445, DOI 10.1146/annurev-physchem-040215-112308, URL https://doi.org/10. 1146/annurev-physchem-040215-112308, pMID: 28301757, https://doi. org/10.1146/annurev-physchem-040215-112308

Cohen AJ, Mori-Sánchez P, Yang W (2008) Insights into current limitations of density functional theory. Science 321(5890):792-794

Dion M, Rydberg H, Schröder E, Langreth DC, Lundqvist BI (2004) Van der Waals density functional for general geometries. Phys Rev Lett 92(24):246,401, DOI 10.1103/PhysRevLett.92.246401, URL http://link.aps.org/doi/10.1103/ PhysRevLett.92.246401

Elliott P, Burke K (2009) Non-empirical derivation of the parameter in the b88 exchange functional. Can J Chem Ecol 87(10):1485-1491, DOI 10.1139/V09-095, URL http: //dx.doi.org/10.1139/V09-095

Engel E, Dreizler RM (2011) Density Functional Theory: An Advanced Course. Springer, Berlin

Erhard J, Bleiziffer P, Görling A (2016) Power series approximation for the correlation kernel leading to Kohn-Sham methods combining accuracy, computational efficiency, and general applicability. Phys Rev Lett 117:143,002, DOI 10.1103/PhysRevLett.117.143002, URL https://link.aps.org/doi/10.1103/PhysRevLett.117.143002 
Ernzerhof M, Scuseria GE (1999) Assessment of the Perdew-Burke-Ernzerhof exchange-correlation functional. J Chem Phys 110:5029, URL http://link . aip. org/link/JCPSA6/v110/i11/p5029/s1

Eshuis $\mathrm{H}$, Yarkony J, Furche $\mathrm{F}$ (2010) Fast computation of molecular random phase approximation correlation energies using resolution of the identity and imaginary frequency integration. The Journal of Chemical Physics 132(23):234114, DOI 10.1063/ 1.3442749, URL http://link.aip.org/link/?JCP/132/234114/1

Flick J, Ruggenthaler M, Appel H, Rubio A (2015) Kohn-Sham approach to quantum electrodynamical density-functional theory: Exact time-dependent effective potentials in real space. Proceedings of the National Academy of Sciences 112(50):15,285-15,290

Furche $F$ (2001) Molecular tests of the random phase approximation to the exchangecorrelation energy functional. Phys Rev B 64:195,120

Furche $\mathrm{F}$ (2008) Developing the random phase approximation into a practical post-KohnSham correlation model. The Journal of Chemical Physics 129(11):114105

Gill PMW, Johnson BG, Pople JA, Frisch MJ (1992) An investigation of the performance of a hybrid of hartree-fock and density functional theory. International Journal of Quantum Chemistry 44(S26):319-331, DOI 10.1002/qua.560440828, URL http: //dx.doi.org/10.1002/qua.560440828

Grimme S (2006) Semiempirical GGA-type density functional constructed with a longrange dispersion correction. Journal of Computational Chemistry 27(15):1787-1799

Gross E, Kohn W (1985) Local density-functional theory of frequency-dependent linear response. Phys Rev Lett 55:2850

Groth S, Dornheim T, Sjostrom T, Malone FD, Foulkes WMC, Bonitz M (2017) Ab initio exchange-correlation free energy of the uniform electron gas at warm dense matter conditions. Phys Rev Lett 119:135,001, DOI 10.1103/PhysRevLett.119.135001, URL https://link.aps.org/doi/10.1103/PhysRevLett.119.135001

Gunnarsson O, Lundqvist B (1976) Exchange and correlation in atoms, molecules, and solids by the spin-density-functional formalism. Phys Rev B 13:4274

Harris J, Jones R (1974) The surface energy of a bounded electron gas. J Phys F 4:1170

Heitler W, London F (1927) Interaction between neutral atoms and homopolar binding according to quantum mechanics. Z Physik 44:455

Hermann J, DiStasio RA, Tkatchenko A (2017) First-principles models for van der waals interactions in molecules and materials: Concepts, theory, and applications. Chemical Reviews 117(6):4714-4758, DOI 10.1021/acs.chemrev.6b00446, URL http://dx. doi.org/10.1021/acs. chemrev.6b00446 pMID: 28272886

Heyd J, Scuseria GE, Ernzerhof M (2003) Hybrid functionals based on a screened coulomb potential. The Journal of Chemical Physics 118(18):8207-8215, DOI 10.1063/ 1.1564060, URL http://link.aip.org/link/?JCP/118/8207/1

J P Perdew SHVKAJMRPDS J A Chevary, Fiolhais C (1992) Atoms, molecules, solids, and surfaces: Applications of the generalized gradient approximation for exchange and correlation. Phys Rev B 46:6671

Janesko BG, Henderson TM, Scuseria GE (2009) Screened hybrid density functionals for solid-state chemistry and physics. Physical Chemistry Chemical Physics 11(3):443454

Johnson ER, Becke AD (2006) Van der waals interactions from the exchange hole dipole moment: Application to bio-organic benchmark systems. Chemical Physics Letters 432(4 - 6):600-603

Johnson ER, Becke AD (2017) Communication: Dft treatment of strong correlation in 3d transition-metal diatomics. The Journal of Chemical Physics 146(21):211,105

Jones R, Gunnarsson O (1989) The density functional formalism, its applications and prospects. Rev Mod Phys 61:689

Jurecka P, Sponer J, Cerny J, Hobza P (2006) Benchmark database of accurate (MP2 and $\mathrm{CCSD}(\mathrm{T})$ complete basis set limit) interaction energies of small model complexes, DNA base pairs, and amino acid pairs. Phys Chem Chem Phys 8:1985-1993 
Klimeš J, Bowler DR, Michaelides A (2009) Chemical accuracy for the van der waals density functional. Journal of Physics: Condensed Matter 22(2):022,201

Kohn W, Sham LJ (1965) Self-consistent equations including exchange and correlation effects. Phys Rev 140(4A):A1133-A1138, DOI 10.1103/PhysRev.140.A1133, URL http://link.aps.org/doi/10.1103/PhysRev.140.A1133

Kümmel S, Kronik L (2008) Orbital-dependent density functionals: Theory and applications. Rev Mod Phys 80(1):3-60, DOI 10.1103/RevModPhys.80.3, URL http://dx. doi.org/10.1103/RevModPhys.80.3

LA Curtiss PR, Raghavachari K (2005) Assessment of gaussian-3 and density-functional theories on the g3/05 test set of experimental energies. J Chem Phys 123:124,107

Langreth D, Mehl M (1981) Phys Rev Lett 47:446

Langreth D, Mehl M (1983) Phys Rev B 28:1809

Langreth D, Perdew J (1975) The exchange-correlation energy of a metallic surface. Solid State Commun 17:1425

Langreth D, Perdew J (1977) Exchange-correlation energy of a metallic surface: Wavevector analysis. Phys Rev B 15:2884

Lee C, Yang W, Parr RG (1988) Development of the colle-salvetti correlation-energy formula into a functional of the electron density. Phys Rev B 37(2):785-789, DOI 10. 1103/PhysRevB.37.785, URL http://link.aps.org/doi/10.1103/PhysRevB. 37.785

Leininger T, Stoll H, Werner HJ, Savin A (1997) Combining long-range configuration interaction with short-range density functionals. Chemical physics letters 275(3-4):151-160

Li L, Baker TE, White SR, Burke K (2016a) Pure density functional for strong correlation and the thermodynamic limit from machine learning. Phys Rev $B$ 94:245,129, DOI 10.1103/PhysRevB.94.245129, URL http://link.aps.org/doi/ 10.1103/PhysRevB.94.245129

Li L, Snyder JC, Pelaschier IM, Huang J, Niranjan UN, Duncan P, Rupp M, Mller KR, Burke $\mathrm{K}(2016 \mathrm{~b})$ Understanding machine-learned density functionals. International Journal of Quantum Chemistry 116(11):819-833, DOI 10.1002/qua.25040, URL http://dx. doi.org/10.1002/qua.25040

M Grüning AM, Rubio A (2006) Density functionals from many-body perturbation theory: The band gap for semiconductors and insulators. J Chem Phys 124:154,108

M Petersilka UG, Gross E (1996) Excitation energies from time-dependent densityfunctional theory. Phys Rev Lett 76:1212

M Stadele JMAG M Moukara, Vogl P (1999) Exact exchange Kohn-Sham formalism applied to semiconductors. Phys Rev B 59:10,031

Ma SK, Brueckner K (1968) Correlation energy of an electron gas with a slowly varying high density. Phys Rev 165:18

Maitra NT (2016) Perspective: Fundamental aspects of time-dependent density functional theory. The Journal of Chemical Physics 144(22):220901, DOI http://dx.doi.org/ 10.1063/1.4953039, URL/http://scitation.aip.org/content/aip/journal/ jcp/144/22/10.1063/1.4953039

Maitra NT, Zhang F, Cave RJ, Burke K (2004) Double excitations within timedependent density functional theory linear response. The Journal of Chemical Physics 120(13):5932-5937, DOI 10.1063/1.1651060, URL http://link.aip.org/link/ ? JCP / $120 / 5932 / 1$

Martin RM, Reining L, Ceperley DM (2016) Interacting Electrons. Cambridge University Press

Medvedev MG, Bushmarinov IS, Sun J, Perdew JP, Lyssenko KA (2017) Response to comment on density functional theory is straying from the path toward the exact functional. Science 356(6337):496-496

Motta M, Ceperley DM, Chan GKL, Gomez JA, Gull E, Guo S, Jiménez-Hoyos CA, Lan TN, Li J, Ma F, Millis AJ, Prokof'ev NV, Ray U, Scuseria GE, Sorella S, Stoudenmire EM, Sun Q, Tupitsyn IS, White SR, Zgid D, Zhang S (2017) Towards the solution of the 
many-electron problem in real materials: Equation of state of the hydrogen chain with state-of-the-art many-body methods. Phys Rev X 7:031,059, DOI 10.1103/PhysRevX.7. 031059, URL https:// link.aps.org/doi/10.1103/PhysRevX.7.031059

Onida G, Reining L, Rubio A (2002) Electronic excitations: density-functional versus many-body green's-function approaches. Rev Mod Phys 74(2):601-659, DOI 10.1103/ RevModPhys.74.601, URL http://dx.doi.org/10.1103/RevModPhys.74.601

P J Stephens CFC F J Devlin, Frisch MJ (1994) J Phys Chem 98:11,623

Perdew J (1985) What do the Kohn-Sham orbitals mean? How do atoms dissociate?, Plenum, NY, p 265

Perdew J (1991) Electronic structure of solids 91, edited by ziesche, p. and eschrig, h.(berlin: Akademie-verlag) p. 11; perdew, jp and wang, y., 1992. Phys Rev B 45(13):244

Perdew J, Wang Y (1986) Accurate and simple density functional for the electronic exchange energy: Generalized gradient approximation. Phys Rev B 33:8800

Perdew JP, Schmidt K (2001) Jacobs ladder of density functional approximations for the exchange-correlation energy. In: AIP Conference Proceedings, AIP, vol 577, pp 1-20

Perdew JP, Wang Y (1992) Accurate and simple analytic representation of the electrongas correlation energy. Phys Rev B 45(23):13,244-13,249, DOI 10.1103/PhysRevB.45. 13244, URL http://link.aps.org/doi/10.1103/PhysRevB.45.13244

Perdew JP, Parr RG, Levy M, Balduz JL (1982) Density-functional theory for fractional particle number: Derivative discontinuities of the energy. Phys Rev Lett 49:16911694, DOI 10.1103/PhysRevLett.49.1691, URL http://link.aps.org/doi/10. 1103 /PhysRevLett.49.1691

Perdew JP, Burke K, Ernzerhof M (1996a) Generalized gradient approximation made simple. Phys Rev Lett 77(18):3865-3868, DOI 10.1103/PhysRevLett.77.3865, URL http: //dx.doi.org/10.1103/PhysRevLett.77.3865, ibid. 78, 1396(E) (1997)

Perdew JP, Ernzerhof M, Burke K (1996b) Rationale for mixing exact exchange with density functional approximations. The Journal of Chemical Physics 105(22):9982-9985, DOl 10.1063/1.472933, URL http://link.aip.org/link/?JCP/105/9982/1

Perdew JP, Yang W, Burke K, Yang Z, Gross EKU, Scheffler M, Scuseria GE, Henderson TM, Zhang IY, Ruzsinszky A, Peng H, Sun J (2017) Understanding band gaps of solids in generalized Kohn-Sham theory. Proceedings of the National Academy of Sciences DOI 10.1073/pnas.1621352114, URL http://www . pnas . org/content/ early/2017/02/28/1621352114.full

Pribram-Jones A, Gross DA, Burke K (2015) DFT: A theory full of holes? Annual Review of Physical Chemistry 66(1):283-304, DOI 10.1146/ annurev-physchem-040214-121420, URL http://www.annualreviews.org/ doi/abs/10.1146/annurev-physchem-040214-121420

Qiu Y, Henderson TM, Zhao J, Scuseria GE (2017) Projected coupled cluster theory. The Journal of Chemical Physics 147(6):064,111

Requist R, Gross E (2016) Exact factorization-based density functional theory of electrons and nuclei. Physical review letters 117(19):193,001

Runge E, Gross EKU (1984) Density-functional theory for time-dependent systems. Phys Rev Lett 52(12):997, DOI 10.1103/PhysRevLett.52.997, URL http: / / link . aps . org/ doi/10.1103/PhysRevLett.52.997

Rupp M, Tkatchenko A, Müller KR, von Lilienfeld OA (2012) Fast and accurate modeling of molecular atomization energies with machine learning. Phys Rev Lett 108:058,301, DOI 10.1103/PhysRevLett.108.058301, URL http:// link.aps.org/doi/10.1103/ PhysRevLett.108.058301

S H Vosko LW, Nusair M (1980) Accurate spin-dependent electron liquid correlation energies for local spin density calculations: a critical analysis. Can J Phys 58(8):1200-1211, DOI 10.1139/p80-159, URL http://www.nrcresearchpress. $\mathrm{com} / \mathrm{doi} / \mathrm{abs} / 10.1139 / \mathrm{p} 80-159$ 
Savin A (1996) On degeneracy, near-degeneracy and density functional theory. Tech. rep., Louisiana State University, Baton Rouge, LA (United States)

Seidl A, Görling A, Vogl P, Majewski JA, Levy M (1996) Generalized Kohn-Sham schemes and the band-gap problem. Phys Rev B 53:3764-3774, DOI 10.1103/PhysRevB.53.3764, URL http://link.aps.org/doi/10.1103/PhysRevB.53.3764

Smith JC, Sagredo F, Burke K (2017) Warming up density functional theory. Submitted URL https://arxiv.org/abs/1701.00873

Snyder JC, Rupp M, Hansen K, Mueller KR, Burke K (2012) Finding density functionals with machine learning. Phys Rev Lett 108:253,002

Snyder JC, Rupp M, Hansen K, Blooston L, Müller KR, Burke K (2013) Orbital-free bond breaking via machine learning. The Journal of Chemical Physics 139(22):224104

Stein T, Kronik L, Baer R (2009) Reliable prediction of charge transfer excitations in molecular complexes using time-dependent density functional theory. Journal of the American Chemical Society 131(8):2818-2820, DOI 10.1021/ja8087482, URL http: // pubs.acs.org/doi/abs/10.1021/ja8087482,http://pubs.acs.org/doi/ $\mathrm{pdf} / 10.1021 / \mathrm{ja} 8087482$

Sun J, Marsman M, Csonka GI, Ruzsinszky A, Hao P, Kim YS, Kresse G, Perdew JP (2011) Self-consistent meta-generalized gradient approximation within the projectoraugmented-wave method. Physical Review B 84(3):035,117

Sun J, Ruzsinszky A, Perdew JP (2015) Strongly constrained and appropriately normed semilocal density functional. Phys Rev Lett 115:036,402, DOI 10.1103/PhysRevLett. 115.036402, URL http://link.aps.org/doi/10.1103/PhysRevLett.115. 036402

Tkatchenko A, Scheffler M (2009) Accurate Molecular Van Der Waals Interactions from Ground-State Electron Density and Free-Atom reference data. Phys Rev Lett 102:073,005

Toulouse J, Gerber IC, Jansen G, Savin A, Ángyán JG (2009) Adiabatic-connection fluctuation-dissipation density-functional theory based on range separation. Phys Rev Lett 102:096,404, DOI 10.1103/PhysRevLett.102.096404, URL https://link.aps. org/doi/10.1103/PhysRevLett.102.096404

Vu K, Snyder JC, Li L, Rupp M, Chen BF, Khelif T, Mller KR, Burke K (2015) Understanding kernel ridge regression: Common behaviors from simple functions to density functionals. International Journal of Quantum Chemistry 115(16):1115-1128, DOI 10.1002/qua.24939, URL http://dx.doi.org/10.1002/qua.24939

Wagner LO, Stoudenmire EM, Burke K, White SR (2013) Guaranteed convergence of the Kohn-Sham equations. Phys Rev Lett 111:093,003, DOI 10.1103/PhysRevLett. 111.093003, URL http://link.aps.org/doi/10.1103/PhysRevLett.111. 093003

Wagner LO, Baker TE, Stoudenmire M E, Burke K, White SR (2014) Kohn-Sham calculations with the exact functional. Phys Rev B 90:045,109, DOI 10.1103/PhysRevB.90. 045109, URL http://link.aps.org/doi/10.1103/PhysRevB.90.045109

Zhao Y, Truhlar D (2008) The m06 suite of density functionals for main group thermochemistry, thermochemical kinetics, noncovalent interactions, excited states, and transition elements: two new functionals and systematic testing of four m06-class functionals and 12 other functionals. Theor Chem Accounts 120:215-241

Zhao Y, Truhlar DG (2006) A new local density functional for main-group thermochemistry, transition metal bonding, thermochemical kinetics, and noncovalent interactions. The Journal of Chemical Physics 125(19):194101, DOI 10.1063/1.2370993, URL http:// link.aip.org/link/?JCP/125/194101/1

Zhao Y, Schultz NE, Truhlar DG (2006) Design of density functionals by combining the method of constraint satisfaction with parametrization for thermochemistry, thermochemical kinetics, and noncovalent interactions. Journal of Chemical Theory and Computation 2(2):364-382 
Zheng X, Cohen AJ, Mori-Sánchez P, Hu X, Yang W (2011) Improving band gap prediction in density functional theory from molecules to solids. Phys Rev Lett 107:026,403, URL http://link.aps.org/doi/10.1103/PhysRevLett.107.026403 